\title{
Software Construction of Experimental Environment for Computer Network Course
}

\author{
Zhi Sun, Liang Xu and Weijun Chen \\ Qingdao Branch, Naval Aeronautical Engineering Institute, Qingdao, China \\ \{sunzhi168 \& xuliang \& chenweijun\}@163.com
}

\begin{abstract}
At present, shortages of network laboratory equipment and funds are the problems of construction in network lab. The sniffer tools, virtual machine, and network simulation software are adopted to build a network experimental environment, thus saving the investment in network laboratory equipment, reducing the network lab managers' burden, and improving the learning effects.

Index Terms - Network Experiment, Network Simulation, Virtual Machine, NS2.
\end{abstract}

\section{Introduction}

At present, "Computer Network" has become a core course for computer specialty and a common course for noncomputer specialties in universities, and almost all universities have opened the related course of computer network principle. This course has strong practicality, involving a lot of abstract theoretical knowledge and network protocols. Therefore, it is hard to vividly and accurately describe and explain the knowledge points in instruction simply by using ordinary courseware and simple animation. The students generally reflected that the network theory course is a bit dull, with unsatisfactory learning effect. Increasing experimental hours properly is an effective solution for the question mentioned above. The students can strengthen their perceptual knowledge by doing the experiment by themselves, and their understanding of the content is also more profound. However, the hardware and equipment involved in network experiments are quite expensive, and a large quantity of laboratory equipments are need, therefore, it will cause some prominent problems ${ }^{[1-3]}$. These problems lead to the result that the experimental hours for the computer network course cannot meet the need of teaching, seriously influencing the learning effect. In order to solve the contradiction, this paper discusses the method to construct the experimental environment for network courses with the help of related types of software. This practice can effectively reduce the cost for laboratory construction and maintenance, and alleviate the workload of laboratory administrative personnel, meanwhile, the students are not limited by experimental location and opening hours. It helps to strengthens the students' perceptual knowledge about what they have learned, and enhance their learning interests and effect.

In learning of the computer network course, the assistant software to be used in experimental classes is mainly divided into network sniffers, network simulators, network emulation analysis and virtual machines. With the aid of such software, most of the experiment contents in network course can be completed in a software experimental environment.

\section{Network Sniffing}

Network sniffing is a kind of network analyzing based on the principle of passive interception. This kind of software mainly monitors, captures and analyzes data packages on the network through network sniffing, and makes various reference data analysis based on them. It is widely applied in such fields as network failure diagnosis, protocol analysis, network performance and network security analysis ${ }^{[4]}$. In this kind of software, the Sniffer Pro released by NetScout Corporation is the most well-known one, which is preferred by specialists in network analysis ${ }^{[4]}$. Wireshark is also a good choice as a tool for network protocol learning, whose function is similar to that of Sniffer Pro, but it is simpler in operation and completely free, suitable for beginners.

In experiments for network courses, the network sniffer tool can be used to learn the format of various protocol data units (PDU) and the working process of the protocol. In network, services are implemented through the network protocol, the data units of different protocols have different encapsulate formats, and each field has different meaning. Understanding profoundly these data unit format and the meaning of each field is helpful for understanding the working process of the protocol.

For instance, the TCP protocol establishes the connection through "three handshakes". To further understand this, we can use Wireshark to get corresponding data from the network for analysis. Fig. 1 shows the messages caught in browsing a website, in which the messages with the serial numbers of 238 , 239, and 240 are the three messages for establishing the connection between the browser and the server. Click on the 238th message, its message format will be displayed in the underneath region, from which the meanings of the fields of the TCP message and the lengths they occupy can be observed. For example, its attribute field SYN is set to 1, indicating that this is a request message for connection establishing.

The protocol messages involved in the network course can all be analyzed in this way, such as the ARP, IP, UDP, HTTP, the DNS protocols. Through capturing and analyzing different kinds of protocol messages, the students' perceptual knowledge can be effectively strengthened. In addition, students with more interests and ability can learn such practical skills as carrying out network failure diagnosis with this software, so as to further improve the students' network application level. 


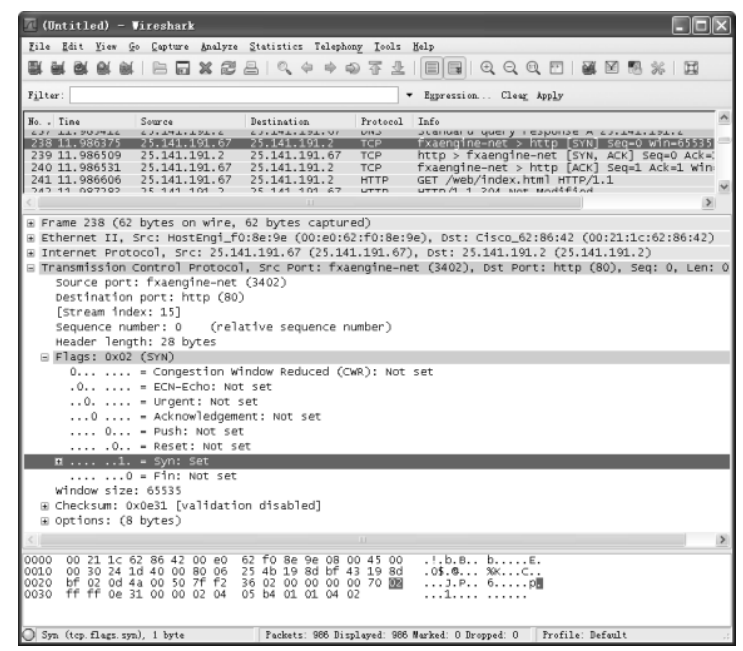

Fig. 1 Data message in Wireshark

\section{Virtual Machine}

The virtual machine technology is a logical resource disposition technology, which is the logic abstract for physical reality. With virtual machine technology, a mainframe can be regarded as several virtual mainframes, and there is no need for the user to think about the actual hardware realization in the background. They only need to transparently run their own systems and software in the virtual environment. Currently in the field of virtual machine technology, the mature products mainly include the VMware series by VMware Corporation, the Virtual PC series by Microsoft. VMware is easy to use, with high operating efficiency, and it provides such network connection patterns as Bridge, NAT, and Host-only, suitable for virtual machine software in network laboratories ${ }^{\left[{ }^{[3}\right.}$.

Usually the network course experiment needs several computers to participate, and sometimes it also uses different operating system platforms, such as local area network construction, network attack and defense experiment, application layer service construction (for example HTTP, FTP, and email). If it is not properly set, it's easy to cause network interruption, and computer system collapse, or even cause network meltdown in the entire laboratory, so as to influence normal conduct of the experiment. For example, when using hacker software to conduct network attack and defense experiment, it's easy to cause network breakdown in the computer laboratory. Install the VMware Workstation virtual machine software on the computer, and use the Bridge mode to simulate one computer into several virtual computers which can conduct network communication. In this environment, the student can carry out certain network experiment between the several virtual machines on the same computer. Under the virtual environment, the students needn't worry about damage of the main system, so they can audaciously carry out all kinds of experiments. Since the virtual experimental environment provides a simulated environment close to reality, therefore the experimental effect will not be influenced, and meanwhile it may reduce the maintenance workload of the network administrator.

\section{Network Simulation}

In order to learn network interconnection and equipment configuring technology, many overseas specialized companies publish various simulators for Cisco experiments, such as RouterSim, and Boson Netsim. The feature of RouterSim is completely designed according to CCNA experiments, quite suitable for learning of CCNA experiment content, however, the network topology of this software cannot be changed, and neither can it be independently designed. However, with the Boson Netsim, we can define network topology according to our need, and we can construct the network at will with Boson Netsim. PC, switch and router can be conveniently and really simulated, therefore this software is usually installed in the network laboratory. Boson Netsim includes two functional modules, respectively the Boson Network Designer and the Boson Netsim. The Boson Network Designer is used to define the network topology, and build the virtual experimental environment, as shown in Fig.2. Boson Netsim provides the IOS mirror images of various equipments, so we can log onto the virtual equipment to carry out configuration ${ }^{[6]}$.

With this software, the students no longer rely on real network equipments. Instead, they can study the configuring commands of switch and router on a single computer. Based on this, they can carry out such experiments as virtual LAN technology, static routing, dynamic routing protocol (RIP, IGRP, OSPF) and access control list (ACL), without complicated cable connection and equipment moving, so that it improves the learning efficiency, and reduces the maintenance workload of the laboratory.

\section{Network Emulation Analysis}

Network emulation analysis is the technology to emulate network behaviors with mathematical modeling and statistical analysis, so as to gain specific network feature parameters. Specifically speaking, network emulation can provide reliable quantitative basis for network planning and designing, validate the actual plan or compare the advantage and disadvantage of several different design plan, simulate various failures in actual network, such as link interruption, or network congestion, and can be used in modeling, validating and analysis of the new network. Network emulation analysis technology has been researched and applied for more than ten years, with such mature products as NS2 and OPNET. OPNET is too expensive to be used in college teaching. NS2 is an open-source free software, anybody can obtain, use and revise its source code, so it is one of the most extensively applied network simulation software in the present network research area. At present NS2 only has Unix and Linux versions, and on the Windows operating system, the Cygwin software can be installed first, which can provide a platform similar to Unix/Linux, and then NS2 can be installed ${ }^{[7]}$. 


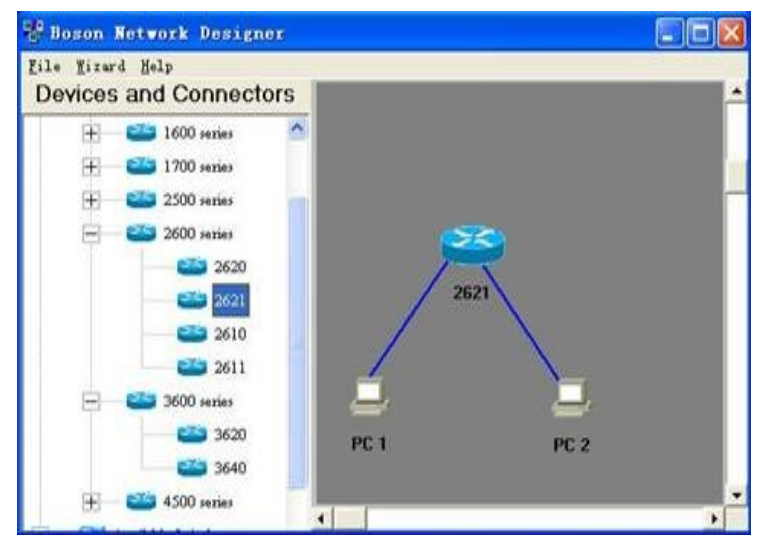

Fig. 2 Self-design network topology with Boson Network Designer

In network course learning, NS2 can be used in the following situations:

1) Some contents which are hard to be expressed but suitable for demonstration with animation can be demonstrated with the NAM animation produced in the process of simulation. For example, when learning the route protocol, the students can observe the change of routing with the change of the network topology through NAM animation. Fig. 3 shows the NAM animation produced in simulating the RIP route protocol with NS2, in which the students can clearly observes that when the network topology changes, the RIP protocol dynamically selects a path with the fewest jumping numbers as the optimal path.

When the paths of the route selected are interrupted (n2 $\mathrm{n} 0, \mathrm{n} 4-\mathrm{n} 8$, and $\mathrm{n} 4-\mathrm{n} 7)$, after the routing table renewal and route computation, the node $\mathrm{n} 3$ will transmit data flow to node $\mathrm{n} 8$ through the new shortest route, as shown in fig.4.

2) Analyze the emulated data produced by NS2, and show the students the result in the form of diagram, and the students will have a visual and quantified view. For instance, with the increase of communication load, when congestion appears in the network, the drop rate and the change of the network throughput can be contrastively analyzed through the diagram of these indices.

3) Students with ability and interest can be guided to

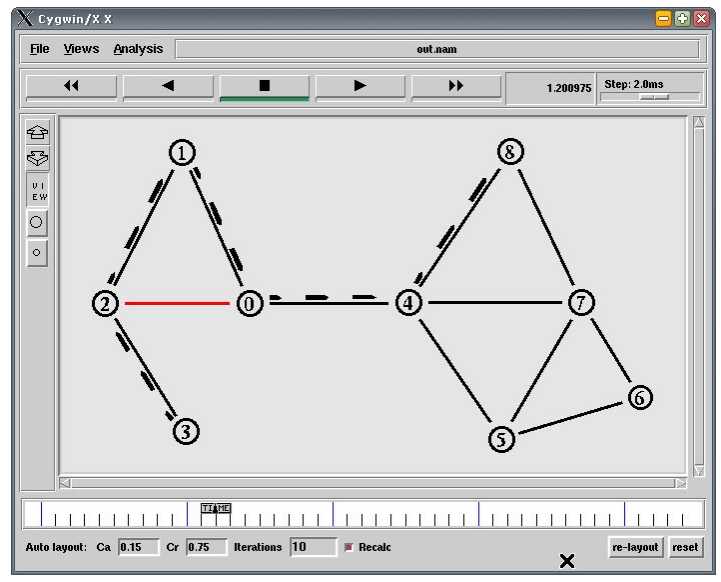

Fig. 3 The initial route selected by RIP protocol

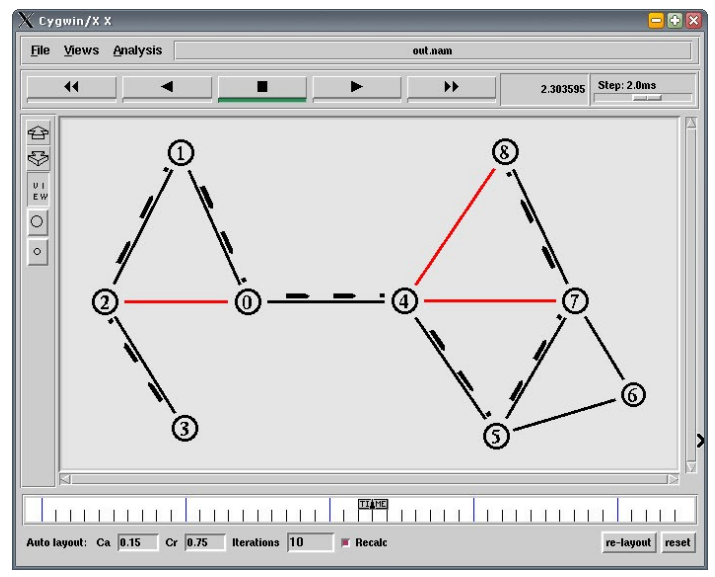

Fig. 4 The new route selected by RIP protocol when topology changed

learn to use NS2. They can carry out emulation analysis on some problems by themselves in learning, which can improve the students' scientific research ability, laying the foundation for their later study and work.

\section{Conclusion}

In computer network course teaching, constructing experimental environment by introducing various softwares can better combine theoretical teaching and practice, which alleviates the problem of insufficient experimental equipments and space, and lowers the probability of equipment damage and the difficulties in laboratory management. In selecting the software used in experiment, we should avoid the practice of pursuing the software with too many functions. Based on the principle of easy operation and economy, software with sufficient functions is selected, which can reduce the performance requirement for computers and the expense for software acquisition. This kind of software can also be recommended to students for installation and learning outside of the laboratory.

\section{References}

[1] Y. Zhou and Y. Li, "Research of network innovation experimental environment based on network virtualization", Acta Electronica Sinica, vol.40, no. 11, pp. 2152-2157, Nov. 2009.

[2] B. Wang and T. Zhang, "Design and implementation of online experimental platform for computer networks course", Computer Education, no. 10, pp. 45-47, May. 2012.

[3] Q. Zhang and B. Zhang, "Application of protocol analysis software in the computer network experiment teaching", Laboratory Science, no. 3, pp. 119-121, May. 2010.

[4] Y. Zhang, H. Sun, H. Qi, "Research and implementation on sniffer detection system in LAN", Network Security Technology \& Application, no. 2, pp. 23-25, 2007.

[5] Q. Liu and J. Li, "Construction of the computer network experiment teaching system". Experimental science and Technology, no.1, pp. 97 99, 2010.

[6] P. Huang and M. Ren, "Constructing the computer network experimental platform based on simulation technology", Experimental science and technology, no. 6, pp. $48-50,2009$.

[7] L. Xu and B. Pang, "NS and network simulation", Beijing: The People's Post Press, 2003. 